\title{
The Anterolateral Ligament of the Knee: What the Radiologist Needs to Know
}

\author{
Pieter Van Dyck, MD, $\mathrm{PhD}^{1}$ Eline De Smet, $\mathrm{MD}^{1}$ Valérie Lambrecht, $\mathrm{MD}^{2}$ \\ Christiaan H. W. Heusdens, MD ${ }^{3}$ Francis Van Glabbeek, MD, PhD ${ }^{3} \quad$ Filip M. Vanhoenacker, MD, PhD $1,2,4$ \\ Jan L. Gielen, MD, PhD ${ }^{1}$ Paul M. Parizel, MD, $\mathrm{PhD}^{1}$ \\ ${ }^{1}$ Department of Radiology, Antwerp University Hospital and \\ University of Antwerp, Edegem, Belgium \\ 2 Department of Radiology, Ghent University Hospital, Ghent, Belgium \\ ${ }^{3}$ Department of Orthopaedics, Antwerp University Hospital and \\ University of Antwerp, Edegem, Belgium \\ ${ }^{4}$ Department of Radiology, AZ Sint-Maarten, Duffel, Belgium

\begin{abstract}
Address for correspondence Pieter Van Dyck, MD, PhD, Department of Radiology, Antwerp University Hospital and University of Antwerp Wilrijkstaat 10, 2650 Edegem, Belgium

(e-mail: pieter.van.dyck@uza.be).
\end{abstract}

Semin Musculoskelet Radiol 2016;20:26-32.

\begin{abstract}
Keywords

- anterolateral ligament

- anterior cruciate ligament rupture

- anterolateral rotatory instability

- magnetic resonance imaging

The anterolateral ligament (ALL) was recently identified as a distinct component of the anterolateral capsule of the human knee joint with consistent origin and insertion sites. Biomechanical studies revealed that the current association between the pivot shift and an injured anterior cruciate ligament (ACL) should be loosened and that the rotational component of the pivot shift is significantly affected by the ALL. This may change the clinical approach toward ACL-injured patients presenting with anterolateral rotatory instability (ALRI), the most common instability pattern after $A C L$ rupture. Radiologists should be aware of the importance of the ALL to ACL injuries. They should not overlook pathology of the anterolateral knee structures, including the ALL, when reviewing MR images of the ACL-deficient knee. In this article, the current knowledge regarding the anatomy, biomechanical function, and imaging appearance of the ALL of the knee is discussed with emphasis on the clinical implications of these findings.
\end{abstract}

\section{The Story Behind}

Even with the most modern anterior cruciate ligament (ACL) reconstruction techniques and in cases without technical errors that feature appropriate tunnel positioning, restoration of rotational stability remains challenging. ${ }^{1}$ Persistent anterolateral rotational instability (ALRI) as revealed by a positive pivot shift test result has been reported in 10 to $30 \%$ of patients after ACL reconstruction. ${ }^{1,2}$ The importance of the anterolateral structures to ACL injuries is not a new finding. Several studies have shown that they work in synergy with the ACL. ${ }^{3}$ Historically, isolated lateral extra-articular tenodesis (LET) was the standard procedure to treat ALRI in ACLdeficient knees ( - Fig. 1). However, because of poor clinical results of LET, these procedures were largely abandoned with the introduction of intra-articular techniques. ${ }^{1,3,4}$
Previous studies have mentioned the existence of a capsuloligamentous structure connecting the femur with the tibia at the anterolateral aspect of the knee joint, but information on the precise anatomy and function of this structure has always been vague and confusing. ${ }^{5-9}$ Already in 1879, the French surgeon Paul Segond described a remarkably constant avulsion fracture at the proximolateral tibia as a result of forced internal tibial rotation applied to cadaveric human knees ${ }^{10}$ (- Fig. 2). However, the anatomical structure responsible for the avulsion of this bony flake from the lateral tibia was not studied in detail. The Segond fracture then became largely neglected in the literature. In 1979, exactly 100 years after the first description of the fracture by Segond, Woods et al correlated the Segond fracture to the presence of significant knee instability and concomitant ACL rupture. ${ }^{11} \mathrm{At}$ the same time, Jack Hughston published his findings on
Issue Theme Knee and Ankle Imaging; Guest Editor, Marco Zanetti, MD
Copyright $\odot 2016$ by Thieme Medical Publishers, Inc., 333 Seventh Avenue, New York, NY 10001, USA. Tel: +1(212) 584-4662.
DOI http://dx.doi.org/ 10.1055/s-0036-1579679. ISSN 1089-7860. 

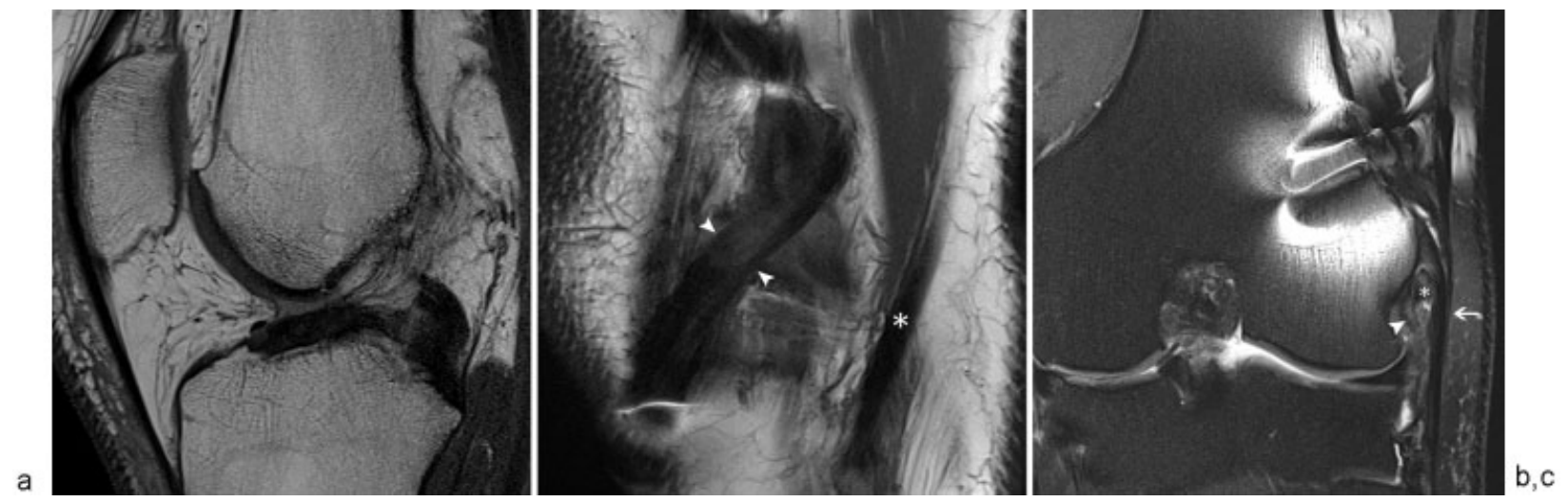

Fig. 1 Nonanatomical lateral extra-articular tenodesis (LET). (a) Sagittal proton-density weighted image (PD-WI) shows chronic anterior cruciate ligament tear. (b) Sagittal PD-WI demonstrates transfer of the distal iliotibial band (arrowheads) to the posterior aspect of lateral femoral condyle (biceps femoris tendon, asterisk). (c) Coronal intermediate-WI shows LET (asterisk) passing in between the lateral collateral ligament (arrow) and popliteal tendon (arrowhead).

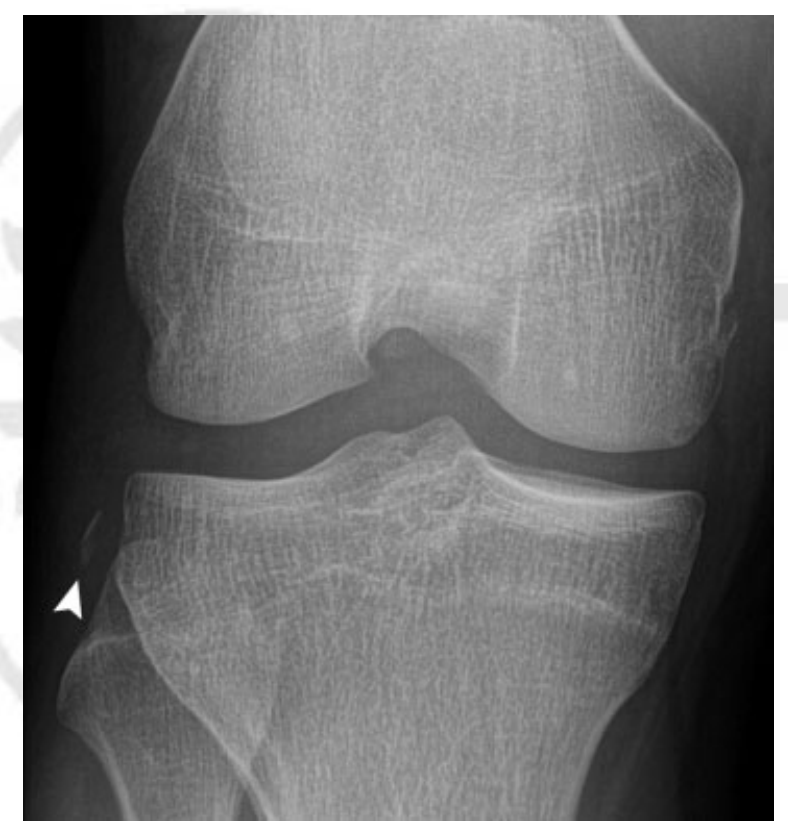

Fig. 2 Segond fracture. Anteroposterior radiograph demonstrating a cortical avulsion fracture (arrowhead) at the proximolateral tibia as a result of forced internal tibial rotation.

rotatory knee instability patterns and described a "mid-third lateral capsular ligament." It was thought to play an important role in the ALRI pattern of the knee, but further anatomical characterization was not provided. Further descriptive articles referenced a ligamentous structure along the anterolateral aspect of the knee that may be responsible for the Segond fracture. ${ }^{6-9}$ A relationship of this structure with the iliotibial band (ITB) or lateral collateral ligament (LCL) was often described ("capsulo-osseous layer of the ITB" by Terry et $\mathrm{al}^{6}$ " "retrograde tract fibers" by Lobenhoffer et al, ${ }^{7}$ and "anterior oblique band of the LCL" by Campos et $\mathrm{al}^{8}$ ). In a detailed anatomical study of the ITB, Terry et $\mathrm{al}^{6}$ in 1986 , and later on, Vieira et $\mathrm{al}^{9}$ in 2007, introduced the term "anterolateral ligament (ALL)" to describe the "capsulo-osseous layer of the ITB." More recently, in 2012, Vincent et al provided a detailed anatomical description of the ALL. ${ }^{12}$ However, it remains unclear whether they found the ALL to be part of the ITB or not. ${ }^{13}$ The publication by Claes et al, ${ }^{13}$ in 2013, was the first to provide a detailed anatomical description of the ALL as a distinct component of the anterolateral capsule.

\section{Anatomy of the ALL}

The ALL runs at the anterolateral aspect of the knee originating from the lateral femoral epicondyle and inserting on the proximal aspect of the anterolateral tibia posterior $(21 \mathrm{~mm})$ to the Gerdy tubercle. ${ }^{12-16}$ Exact anatomical descriptions slightly vary in the literature, which is likely the reason for the variability in reported incidence of the ALL, ranging between $80 \%$ and $100 \%$. Claes et $\mathrm{al}^{13}$ described the femoral attachment site as anterior and distal to the insertion of the LCL, whereas Dodds et $\mathrm{al}^{16}$ found that the femoral insertion site was proximal and posterior to the lateral epicondyle. Caterine et $\mathrm{al}^{15}$ found the femoral insertion site to be located either anterior-distal or posteriorproximal to the insertion site of the LCL. In all the studies, the femoral insertion site of the ALL was found to be posterior and proximal to the origin of the popliteus tendon. In contrast, Vincent et $\mathrm{al}^{12}$ described the origin of the ALL to be on the lateral femoral condyle, just anterior to the popliteus tendon insertion and blending with its fibers. These inconsistent findings may be attributed to difficulties in separating the femoral insertion sites of the ALL and LCL or to the existence of anatomical variants. A cadaveric knee dissection at our institution found the origin of the ALL to be posterior and proximal to the origin of the LCL ( - Fig. 3). Given the fact that the femoral origins of the ALL and LCL are so closely associated, the term "lateral collateral ligament complex" was proposed. In this view, the ALL can be regarded as the lateral counterpart of the deep medial collateral ligament.

The ALL further shows an oblique intracapsular course in layer 3 according to the classification by Seebacher et al ${ }^{17}$ and is clearly distinguishable from the remainder of the lateral capsule. ${ }^{12-16}$ It has a strong connection with the periphery of the body of the lateral meniscus by way of its meniscofemoral and meniscotibial components. In between the ALL and the body of the lateral meniscus, the lateral inferior geniculate 
a
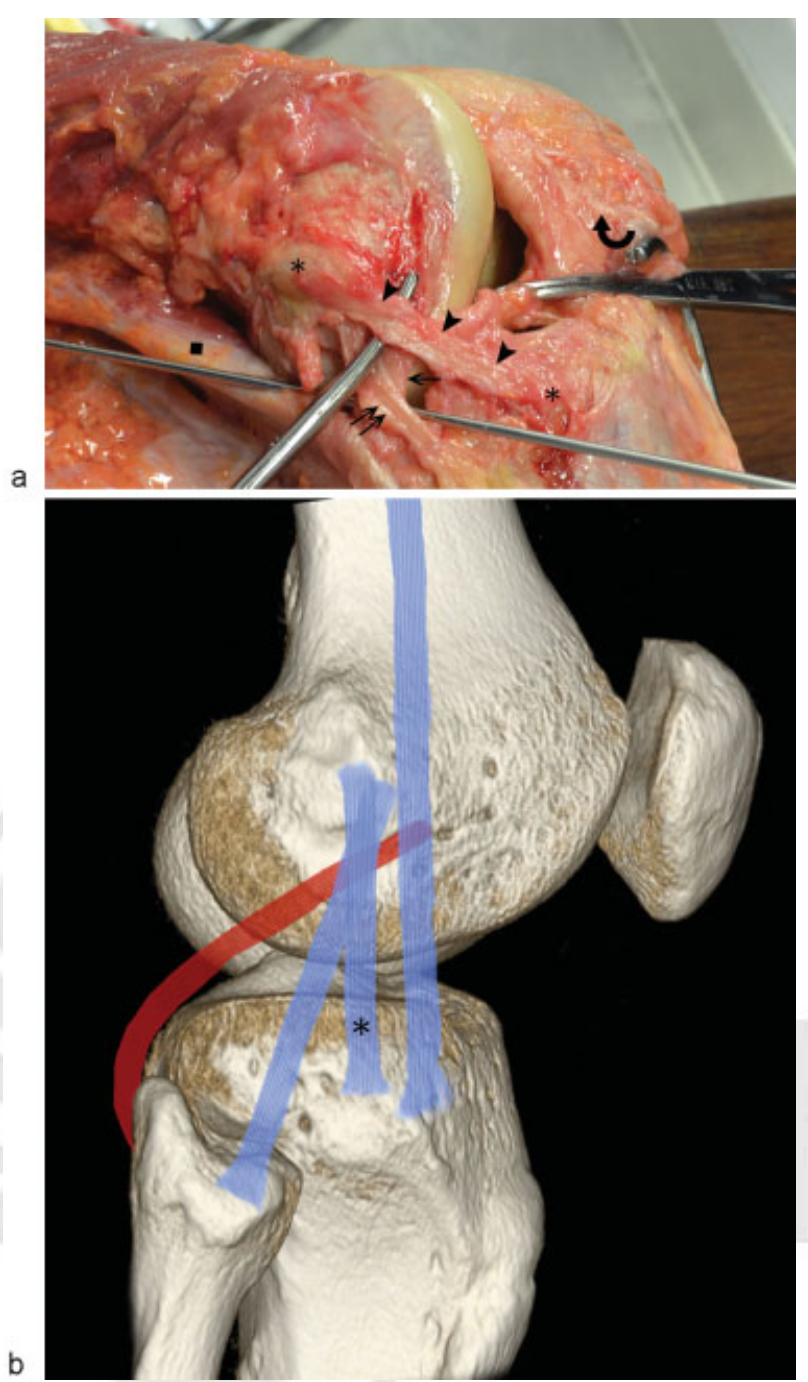

Fig. 3 Anatomy of the anterolateral ligament (ALL). (a) Lateral view of a right knee during dissection. The ALL is denoted in relationship to surrounding structures. With the iliotibial band reflected (curved arrow), the ALL (arrowheads) is clearly distinguishable originating at the lateral femoral epicondyle and inserting to the anterolateral tibia (asterisk). Note fibers at the origin of the ALL to be posterior and proximal to the origin of the lateral collateral ligament (LCL) (double arrow) and the popliteus tendon (arrow). Also note biceps femoris (black box) attaching to the fibular head. (b) Volume computed tomography image with anatomical drawings of the lateral supporting structures. Note close relation of ALL and LCL origin. The ALL (asterisk) inserts midway between the Gerdy tubercle and the fibular head. Popliteus tendon is colored in red.

artery and vein are invariably found. Distally, the ALL inserts approximately midway between the center of the Gerdy tubercle and the anterior margin of the fibular head, proximal and anterior to the anterior arm of the short head of the biceps femoris tibial attachment. It thus forms a capsuloligamentous insertional fold, termed the "lateral tibial recess." There is no connection at all with the distal ITB.

The mean length of the ALL is $41 \mathrm{~mm}$ measured in 90 degrees of flexion and $38 \mathrm{~mm}$ in extension, illustrating some tensioning of the ligament during flexion. ${ }^{13}$ The mean width of the femoral origin measures $8 \mathrm{~mm}$, slightly narrows near the level of the joint line $(7 \mathrm{~mm})$, and then broadens further distally, inserting to the proximal tibia with a width of $11 \mathrm{~mm}$. The average distance between the proximal edge of the lateral tibia and the ALL tibial insertion site is 6 to $9 \mathrm{~mm}$. The mean thickness of the ALL at the level of the joint line is $1.3 \mathrm{~mm} .{ }^{13,18-20}$

\section{Biomechanics of the ALL}

In 2007, Monaco et al compared double-bundle reconstructions and single-bundle associated with LET and concluded that patients with LET exhibited less rotational instability than did those with double-bundle reconstruction. ${ }^{4}$ Later on, in 2012, these researchers studied the function of the anterolateral capsule with the pivot shift test, and they showed that damage to this structure significantly increased rotator knee instability. ${ }^{3}$

With the recent identification of the ALL, several studies have investigated its biomechanical function. ${ }^{18-21}$ These studies revealed that the ALL is an important stabilizer against internal tibial rotation and controls anterolateral laxity, especially at deep flexion angles (35-90 degrees). ${ }^{18-21}$ In contrast, the restraining effect of both the native and reconstructed ACL on tibial rotation is negligible. ${ }^{22}$ Thus an ALL injury significantly affects the pivot shift in the ACL-deficient knee. Moreover, it is possible that a positive pivot shift sign may be observed in some patients with an intact ACL but with (unrecognized) damage to the ALL. The results of these studies yield new insights for diagnosing and treating ALRI, the most common clinical instability pattern after ACL rupture, previously attributed to (posterolateral bundle) injuries of the ACL. ${ }^{2-4,22}$ Thus it has become clear that preoperative judgment of the ALL may be important. However, clinical testing of rotatory laxity is subjective, ${ }^{2}$ and arthroscopy only may provide indirect evidence of ALL injury. ${ }^{23}$ It seems obvious that the ability of MRI to detect the ALL and its eventual injuries needs our attention.

\section{Imaging of the ALL}

Milch et $\mathrm{al}^{24}$ was the first to report the radiologic aspect of the Segond fracture. He described the radiologic findings as an "avulsion of the ITB at its insertion behind Gerdy's tubercle." Recently, a direct link between the ALL tibial attachment and Segond fracture was found, and thus the latter should be seen as the tip of the iceberg, as it were, for lesions of the ALL. ${ }^{22,25}$ This finding suggests that, similar to the clinical significance of the Segond fracture, any ALL lesion should be seen as an important indicator of internal derangement. ${ }^{25,26}$

On MRI, the ALL is best identified in the coronal plane. ${ }^{27}$ Proton density-weighted sequences and T2-weighted sequences with fat saturation are superior to T1-weighted sequences in assessing the ALL. ${ }^{27,28}$ The normal ALL can be followed as a continuous band of low signal intensity from the lateral femoral epicondyle to the anterolateral tibia. ${ }^{27-31} \mathrm{~A}$ discrete proximal origin of the ALL is difficult to discern due to its close association with the LCL, and at this level, the ALL arises anterior to the LCL origin. As it continues distally, deep to the ITB, there is a more distinct meniscotibial component inserting below the level of the lateral tibial plateau and midway between the fibular head and Gerdy tubercle (-Fig. 4). On MRI, the distal insertion may be inseparable anteriorly from the posterior fibers of the ITB. In most cases, MRI clearly 
a
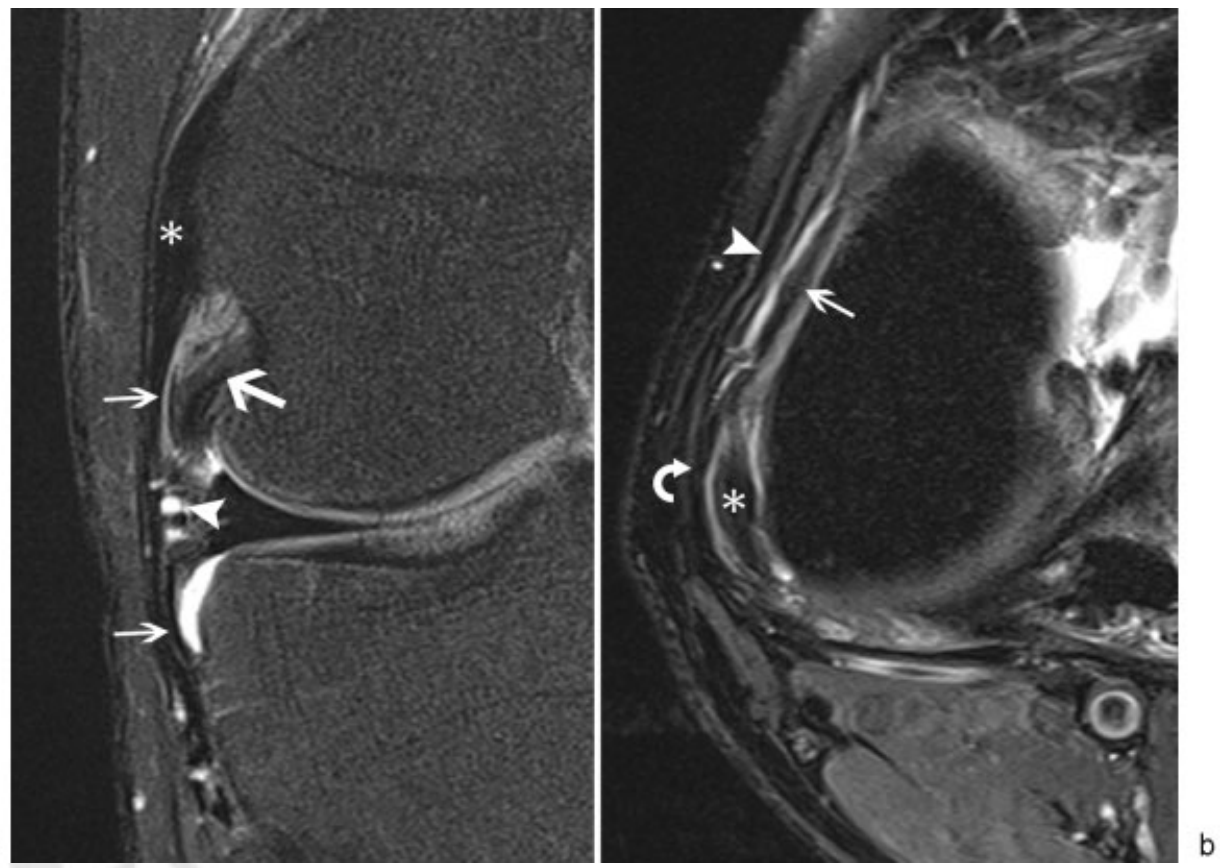

Fig. 4 MR appearance of the normal anterolateral ligament (ALL). (a) Coronal intermediate-weighted image (WI) with fat suppression (fs) shows the normal ALL (small arrows) originating at the lateral femoral epicondyle (asterisk) and inserting to the anterolateral tibia. Note firm attachments of the ALL to the body of the lateral meniscus, proximally and distally from the lateral inferior geniculate vessels (arrowhead). Popliteus tendon, large arrow. (b) Axial fs intermediate-WI shows ALL (arrow) anterior to the lateral collateral ligament (curved arrow), and deep to the iliotibial band (arrowhead). Popliteus tendon, asterisk.

demonstrates attachments of the ALL to the lateral meniscus, separating the ligament in meniscofemoral and meniscotibial portions. The lateral inferior geniculate artery and vein, in between the ALL bifurcation point and the body of the lateral meniscus, can be used as an anatomical reference point and may be helpful to better characterize the ligament.
Several studies have evaluated the ability of MRI to identify the ALL in the uninjured knee. ${ }^{21,27-30}$ These have reported detection rates of the ALL ranging between $72 \%$ and $93 \%$. It is possible that the articular distension associated with an acute knee injury would make it much easier to visualize the ALL on MRI. $^{27,28}$
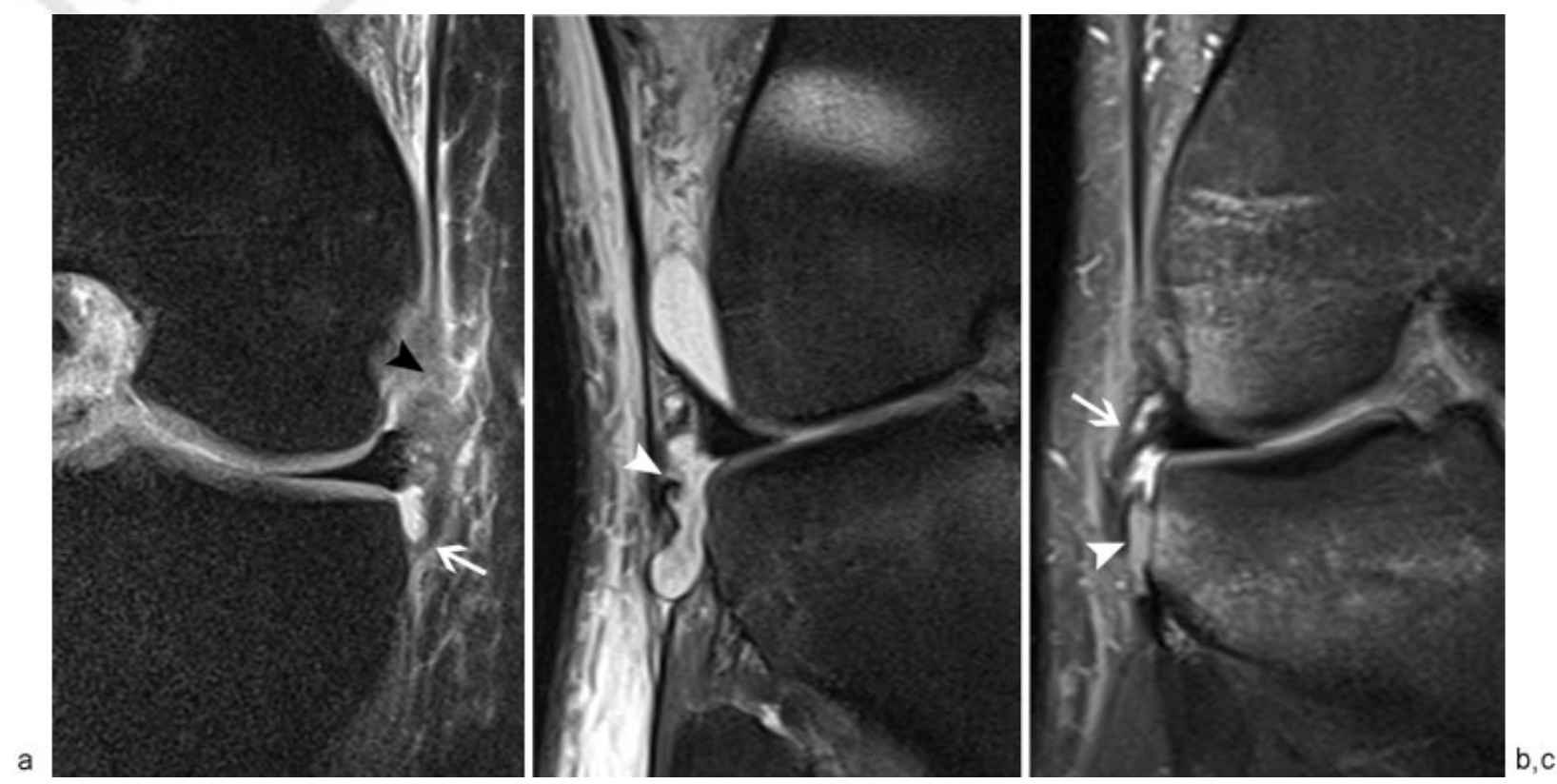

Fig. 5 MR appearance of the abnormal anterolateral ligament (ALL). (a) Coronal fat suppression (fs) intermediate-weighted image shows proximal lesion (arrowhead). Distal part of the ALL is intact (arrow). (b) Distal lesion of the ALL (arrowhead). (c) Bony avulsion of the ALL (arrowhead). Proximal part of the ALL is intact (arrow). All patients had arthroscopically confirmed anterior cruciate ligament (ACL) tear. 
a
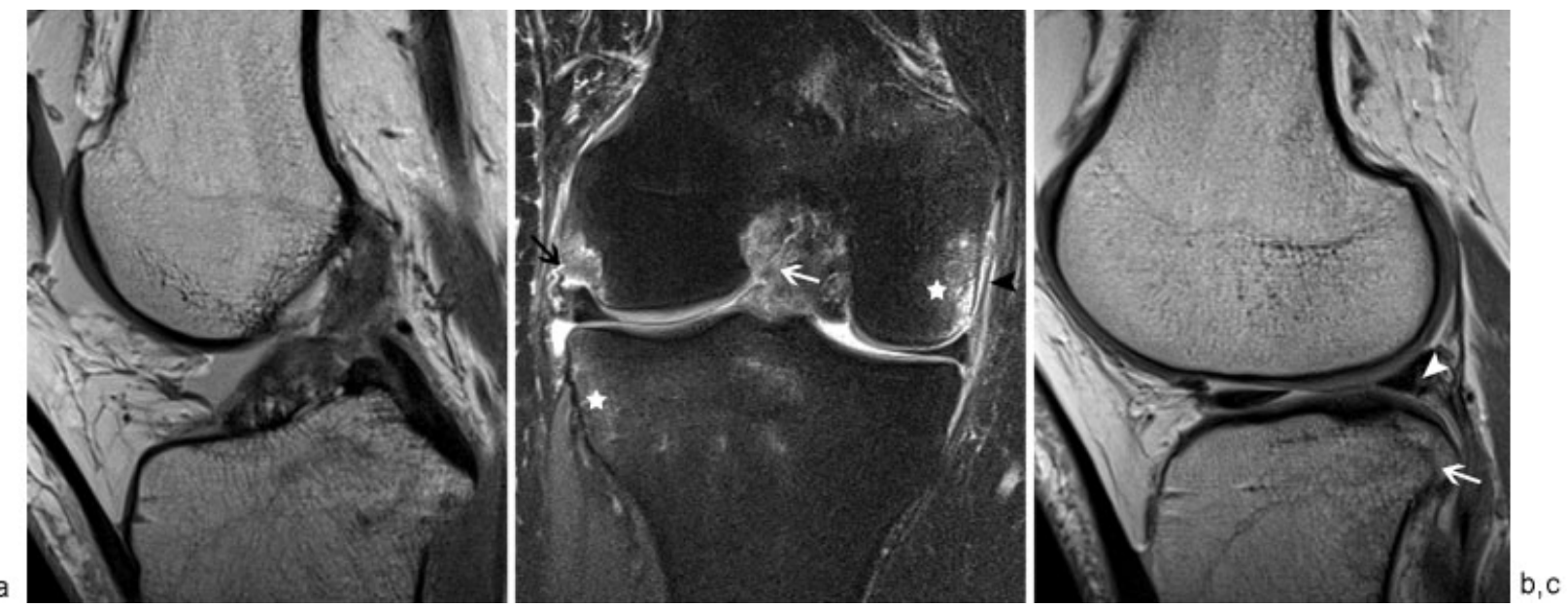

Fig. 6 A 31-year-old woman sustaining an acute knee injury. (a) Sagittal proton density-weighted image (PD-WI) shows complete tear of the anterior cruciate ligament ( $\mathrm{ACL}$ ) and large synovial effusion. (b) Coronal fat suppression (fs) intermediate-WI shows combined $A C L$ (white arrow) and proximal ALL (black arrow) tear. Note extensive bone contusions at the lateral and medial knee compartment (star), and associated sprain of the medial collateral ligament (arrowhead). (c) Sagittal PD-WI through the lateral knee compartment shows peripheral longitudinal tear in the posterior horn of the lateral meniscus (arrowhead). Also note cortical depression fracture at the posterolateral tibia (arrow).
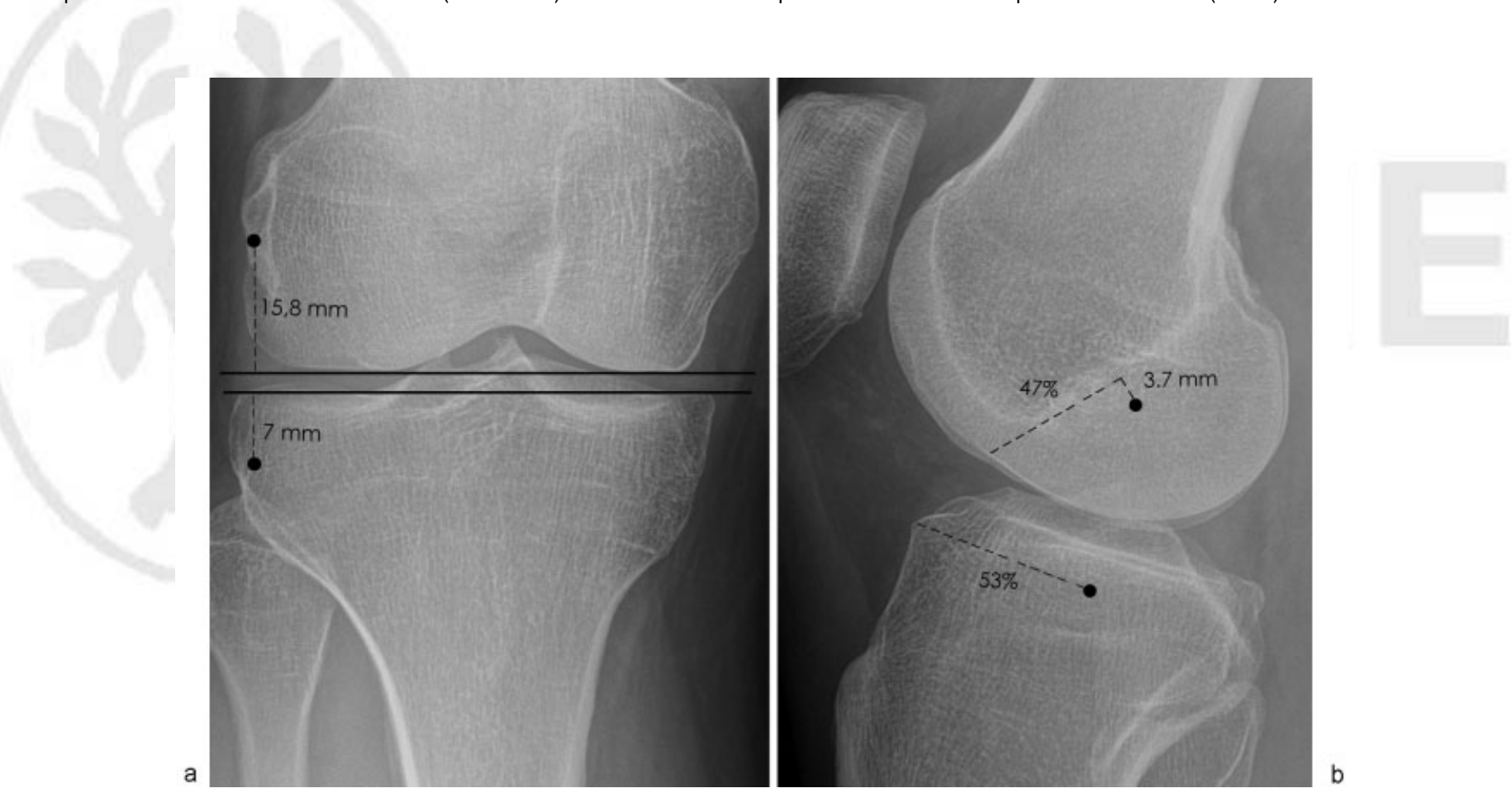

Fig. 7 Radiographic landmarks of the anterolateral ligament (ALL). (a) On anteroposterior radiography, the ALL is located 15.8 mm from the posterior bicondyle line and $7 \mathrm{~mm}$ from the lateral tibial joint line. (b) On the lateral view, the ALL femoral attachment is located $\sim 47 \%$ from the anterior edge of the condyle and $3.7 \mathrm{~mm}$ caudal to Blumensaat line, and the tibial attachment is located $\sim 53 \%$ from the anterior edge of the tibial plateau. ${ }^{39}$

Radiologists must specifically assess the anterolateral knee structures, including the ALL, when reviewing MR images of the ACL-deficient knee (-Figs. 5 and $\mathbf{6}$ ). ACL ruptures most often result from twisting noncontact injuries that have a pivot shift-like mechanism, consisting of anterior tibial subluxation and excess internal tibial rotation. ${ }^{32}$ Thus, given the previously mentioned association between the ALL and the pivot shift, one may hypothesize that an ACL injury might often be accompanied by a concomitant ALL injury. 22

To date, only one previous study, by Claes et al, ${ }^{33}$ described the appearance of the ALL on preoperative MRI in a large consecutive series of ACL reconstructed knees. They considered the ALL to be abnormal on MRI in case of complete disruption, if the contour of the ALL was irregular, if intra- or periligamentous edema existed, or a combination of these features was observed. Furthermore, abnormalities were classified as proximal (above the meniscus) or distal (below the meniscus). Of the 206 patients included, 44 (21.3\%) were considered uninjured; 162 knees (78.8\%) demonstrated radiologic ALL abnormalities. Most of the ALL abnormalities were situated in the distal part of the ligament (77.8\%). These results, however, have not yet been confirmed by other 
authors. Moreover, further research is needed to establish the clinical significance of associated ALL damage on MRI in the ACL-deficient knee.

\section{Therapeutic Implications}

Recent advances in our understanding of the anatomical, biomechanical, and radiologic characteristics of the ALL have led to a resurgent interest in the reconstruction of this structure as part of the management of knee instability. The poor results of early isolated LET could now be explained by a combination of imperfectly anatomical ACL reconstruction, an empirical nonanatomical extra-articular reconstruction, and a postoperative protocol involving immobilization of the knee and a slow rehabilitation program. ${ }^{34}$ In a recent metaanalysis of LET techniques, Hewison et $\mathrm{al}^{35}$ showed a statistically significant reduction in pivot shift in favor of the combined ACL and LET procedure.

With recent studies having characterized the anatomy of the ALL as a distinct ligamentous structure, an additional anatomical reconstruction of the ALL, concomitant to the ACL, is gaining popularity among knee surgeons. ${ }^{36-38}$ Indications for a combined procedure are grade 3 pivot shift, associated Segond fracture amendable for primary refixation, chronic ACL lesion, high level of sporting activity, pivoting sports, and revision ACL surgery cases, especially those without an apparent cause of failure. ${ }^{22,36-38}$ ALL reconstruction can be performed with hamstring tendons or InternalBrace (Arthrex, Naples, FL, USA) augmentation, fixated with anchors while respecting the bony landmarks of the ALL on radiographic images $^{39}$ (-Fig. 7).

Sonnery-Cottet et $\mathrm{al}^{36}$ recently reported on the outcome of a combined ACL and ALL reconstruction technique with a minimum 2-year follow-up in a total of 92 patients. They concluded that a combined reconstruction can be an effective procedure allowing good anteroposterior and rotational laxity control without specific complications such as stiffness or limited range of motion. However, longer term and comparative studies are required to determine whether these combined reconstructions improve the results of ACL surgical outcome.

\section{Conclusion}

1. Despite satisfactory clinical results, intra-articular ACL reconstructions often do not restore normal kinematics and biomechanics of the knee, and they particularly do not fully control knee rotational instability.

2. The anterolateral structures of the knee work in synergy with the ACL and are an important restraint to internal rotation of the lateral compartment. They are often injured during ACL rupture.

3. Historically, anatomical descriptions of the anterolateral knee structures have been vague and confusing. This has led to confusion and difficulty in achieving an anatomical reconstruction of these structures.

4. The ALL was recently identified as a distinct ligamentous structure on the anterolateral aspect of the knee.
Biomechanical studies have shown a high correlation between the pivot shift and an injury to the ALL.

5. Radiologists should assess the anterolateral knee structures, including the ALL, when reviewing MR images of the ACL-deficient knee. Further research is needed to optimize current clinical MR protocols to assess the ALL.

6. Although the first clinical results of combined anatomical ACL and ALL reconstructions are encouraging, long-term follow-up studies are necessary to determine whether these combined reconstructions improve the results of surgical ACL treatment.

\section{References}

1 Murawski CD, van Eck CF, Irrgang JJ, Tashman S, Fu FH. Operative treatment of primary anterior cruciate ligament rupture in adults. J Bone Joint Surg Am 2014;96(8):685-694

2 Lopomo N, Zaffagnini S, Amis AA. Quantifying the pivot shift test: a systematic review. Knee Surg Sports Traumatol Arthrosc 2013; 21(4):767-783

3 Monaco E, Ferretti A, Labianca L, et al. Navigated knee kinematics after cutting of the ACL and its secondary restraint. Knee Surg Sports Traumatol Arthrosc 2012;20(5):870-877

4 Monaco E, Labianca L, Conteduca F, De Carli A, Ferretti A. Double bundle or single bundle plus extraarticular tenodesis in ACL reconstruction? A CAOS study. Knee Surg Sports Traumatol Arthrosc 2007;15(10):1168-1174

5 Hughston JC, Andrews JR, Cross MJ, Moschi A. Classification of knee ligament instabilities. Part II. The lateral compartment. J Bone Joint Surg Am 1976;58(2):173-179

6 Terry GC, Hughston JC, Norwood LA. The anatomy of the iliopatellar band and iliotibial tract. Am J Sports Med 1986;14(1):39-45

7 Lobenhoffer P, Posel P, Witt S, Piehler J, Wirth CJ. Distal femoral fixation of the iliotibial tract. Arch Orthop Trauma Surg 1987; 106(5):285-290

8 Campos JC, Chung CB, Lektrakul N, et al. Pathogenesis of the Segond fracture: anatomic and MR imaging evidence of an iliotibial tract or anterior oblique band avulsion. Radiology 2001; 219(2):381-386

9 Vieira EL, Vieira EA, da Silva RT, Berlfein PA, Abdalla RJ, Cohen M. An anatomic study of the iliotibial tract. Arthroscopy 2007;23(3): 269-274

10 Segond P. Recherches cliniques et expérimentales sur les épanchements sanguins du genou par entorse. Paris, France: Aux Bureaux du Progrès Médical 1879:1-85. Available at: http:// wwwpatrimoineedilivrecom/

11 Woods GW, Stanley RF, Tullos HS. Lateral capsular sign: x-ray clue to a significant knee instability. Am J Sports Med 1979;7(1):27-33

12 Vincent JP, Magnussen RA, Gezmez F, et al. The anterolateral ligament of the human knee: an anatomic and histologic study. Knee Surg Sports Traumatol Arthrosc 2012;20(1):147-152

13 Claes S, Vereecke E, Maes M, Victor J, Verdonk P, Bellemans J. Anatomy of the anterolateral ligament of the knee. J Anat 2013; 223(4):321-328

14 Stijak L, Bumbaširević M, Radonjić V, et al. Anatomic description of the anterolateral ligament of the knee. Knee Surg Sports Traumatol Arthrosc 2014

15 Caterine S, Litchfield R, Johnson M, Chronik B, Getgood A. A cadaveric study of the anterolateral ligament: re-introducing the lateral capsular ligament. Knee Surg Sports Traumatol Arthrosc 2015;23(11):3186-3195

16 Dodds AL, Halewood C, Gupte CM, Williams A, Amis AA. The anterolateral ligament: anatomy, length changes and association with the Segond fracture. Bone Joint J 2014;96-B(3):325-331

17 Seebacher JR, Inglis AE, Marshall JL, Warren RF. The structure of the posterolateral aspect of the knee. J Bone Joint Surg Am 1982;64(4): 536-541 
18 Zens M, Niemeyer P, Ruhhammer J, et al. Length changes of the anterolateral ligament during passive knee motion. A human cadaveric study. Am J Sports Med 2015;43(10):2545-2552

19 Parsons EM, Gee AO, Spiekerman C, Cavanagh PR. The biomechanical function of the anterolateral ligament of the knee. Am J Sports Med 2015;43(3):669-674

20 Kennedy MI, Claes S, Fuso FA, et al. The anterolateral ligament. An anatomic, radiographic, and biomechanical analysis. Am J Sports Med 2015;43(7):1606-1615

21 Van der Watt L, Khan M, Rothrauff BB, et al. The structure and function of the anterolateral ligament of the knee: a systematic review. Arthroscopy 2015;31(3):569-82.e3

22 Claes $\mathrm{S}$. The anterolateral ligament of the knee [PhD dissertation]. Leuven, Belgium: Catholic University of Leuven; 2013

23 LaPrade RF, Gilbert TJ, Bollom TS, Wentorf F, Chaljub G. The magnetic resonance imaging appearance of individual structures of the posterolateral knee. A prospective study of normal knees and knees with surgically verified grade III injuries. Am J Sports Med 2000;28(2):191-199

24 Milch H. Cortical avulsion fracture of the lateral tibial condyle. J Bone Joint Surg Am 1936;18:159-164

25 Claes S, Luyckx T, Vereecke E, Bellemans J. The Segond fracture: a bony injury of the anterolateral ligament of the knee. Arthroscopy 2014;30(11):1475-1482

26 Hess T, Rupp S, Hopf T, Gleitz M, Liebler J. Lateral tibial avulsion fractures and disruptions to the anterior cruciate ligament. A clinical study of their incidence and correlation. Clin Orthop Relat Res 1994;(303):193-197

27 Helito CP, Helito PV, Costa HP, et al. MRI evaluation of the anterolateral ligament of the knee: assessment in routine 1.5-T scans. Skeletal Radiol 2014;43(10):1421-1427

28 Taneja AK, Miranda FC, Braga CA, et al. MRI features of the anterolateral ligament of the knee. Skeletal Radiol 2015;44(3): 403-410

29 Porrino J Jr, Maloney E, Richardson M, Mulcahy H, Ha A, Chew FS. The anterolateral ligament of the knee: MRI appearance, association with the Segond fracture, and historical perspective. AJR Am J Roentgenol 2015;204(2):367-373
30 Kosy JD, Mandalia VI, Anaspure R. Characterization of the anatomy of the anterolateral ligament of the knee using magnetic resonance imaging. Skeletal Radiol 2015;44(11):1647-1653

31 De Maeseneer M, Boulet C, Willekens I, et al. Segond fracture: involvement of the iliotibial band, anterolateral ligament, and anterior arm of the biceps femoris in knee trauma. Skeletal Radiol 2015;44(3):413-421

32 Oh YK, Lipps DB, Ashton-Miller JA, Wojtys EM. What strains the anterior cruciate ligament during a pivot landing? Am J Sports Med 2012;40(3):574-583

33 Claes S, Bartholomeeusen S, Bellemans J. High prevalence of anterolateral ligament abnormalities in magnetic resonance images of anterior cruciate ligament-injured knees. Acta Orthop Belg 2014;80(1):45-49

34 Dodds AL, Gupte CM, Neyret P, Williams AM, Amis AA. Extraarticular techniques in anterior cruciate ligament reconstruction: a literature review. J Bone Joint Surg Br 2011;93(11):1440-1448

35 Hewison CE, Tran MN, Kaniki N, Remtulla A, Bryant D, Getgood AM. Lateral extra-articular tenodesis reduces rotational laxity when combined with anterior cruciate ligament reconstruction: a systematic review of the literature. Arthroscopy 2015;31(10):2022-2034

36 Sonnery-Cottet B, Thaunat M, Freychet B, Pupim BHB, Murphy CG Claes $\mathrm{S}$. Outcome of a combined anterior cruciate ligament and anterolateral ligament reconstruction technique with a minimum 2-year follow-up. Am J Sports Med 2015;43(7):1598-1605

37 Mackay GM, Blyth MJ, Anthony I, Hopper GP, Ribbans WJ. A review of ligament augmentation with the InternalBrace ${ }^{\mathrm{TM}}$ : the surgical principle is described for the lateral ankle ligament and ACL repair in particular, and a comprehensive review of other surgical applications and techniques is presented. Surg Technol Int 2015;26:239-255

38 Helito CP, Bonadio MB, Gobbi RG, et al. Combined intra- and extraarticular reconstruction of the anterior cruciate ligament: the reconstruction of the knee anterolateral ligament. Arthrosc Tech 2015;4(3):e239-e244

39 Helito CP, Demange MK, Bonadio MB, et al. Radiographic landmarks for locating the femoral origin and tibial insertion of the knee anterolateral ligament. Am J Sports Med 2014;42(10):2356-2362 